\title{
Electrochemical Impedance Spectroscopy of Hybrid Epoxy Resin Emulsion Coatings
}

\author{
Rakesh N. Patil, Brihbihari V. Sharma, Prakash A. Mahanwar \\ Department of Polymer \& Surface Engineering, Institute of Chemical Technology, Mumbai, India \\ Email: rakeshpatil1508@gmail.com
}

Received May 9, 2012; revised June 13, 2012; accepted June 25, 2012

\begin{abstract}
A hybrid epoxy resin one pack emulsion with acrylate was synthesized for application in the field of corrosion protection. Electrochemical impedance tests were applied to steel specimens coated with hybrid epoxy and tested after immersion in $3.5 \% \mathrm{NaCl}$ solution at room temperature. Results taken from Nyquist and Bode plots as were analyzed by means of software provided with the instrument. Specimens were examined under scanning electron microscope shows a clear rupture and degradation in hybrid epoxy coating after prolonged exposer to salt solution.
\end{abstract}

Keywords: Hybrid; Epoxy; Electrochemical Impedance Spectroscopy; Coatings

\section{Introduction}

Epoxy resins have excellent characteristic property of corrosion resistance [1]. They have been used as two pack systems in combination of different curing agents as protective coatings [2]. However; they have poor or low fracture energy, high shrinkage, and show brittle behavior [3]. To overcome this disadvantages hybrid of epoxy resin was synthesized with acrylate monomers using emulsion polymerization technique. The hybrid can be defined as, the system synthesized from two or more participating components having superior properties then both the participants. The hybrid epoxy resin synthesized emulsion obtained was one pack system with longer shelf life. For the hybrid to be used as a protective coating, corrosion resistance properties must be satisfactory.

Electrochemical Impedance Spectroscopy (EIS) has many advantages in comparison with other electrochemical techniques. It is a non-destructive method for the evaluation of a wide range of materials, including coatings, anodized films and corrosion inhibitors. It can also provide detailed information of the systems under examination; parameters such as corrosion rate, electrochemical mechanisms and detection of localized corrosion. Polymer based coatings use barrier technology to protect substrates from corrosive chemicals and environments, particularly when in immersion service [4].

This paper reports, results of an investigation of the corrosion resistance of the hybrid coatings using EIS techniques. The hybrid one pack epoxy system was synthesized with conventional emulsion polymerization technique. The effects of coating thickness on water absorbance and ultimately on corrosion resistance were also studied. Nyquist and bode plots was analyzed to predict the corrosion performance of hybrid coatings. SEM analysis of the specimen was performed after immersion to get a closer view of coating degradation.

\section{Experimental}

\subsection{Materials}

Diglycidyl ethers of bisphenol A (DGEBA) epoxy resin (D.E.R. 336) Kindly provided by Dow Chemical international, India. $n$-butylacrylate (BA) and methylmethacrylate (MMA) were used as the acrylic monomers. Acrylic acid (AA) and 2-hydroxyl ethyl methacrylate (HEMA) were used as the functional acrylic monomers purchased from Indofil chemicals, Potassium persulfate (KPS) used as initiator, Ethylene diamine (EDA) solution used for neutralisation and sodium bicarbonate (SBC) as a buffer was purchased from Sigma Aldrich, Nonyl phenoxy polyethyleneoxy ethanol nonionic surfactant HLB 17.8 (Neoigen DK X 405) nonionic surfactant and sodium dodecyl benzene sulfonate (Daninol 25P) were used as anionic surfactant supplied by Dai-Ichi Karkaria. Distilled and Deionized water was used throughout all experiments arranged here. All the other chemicals used in this work were AR grades obtained from a S.D. Fines Chemicals used without further purification.

\subsection{Preparation of Hybrid Epoxy Resin Emulsion}

The synthesis of hybrid emulsion was carried out in a 
$500 \mathrm{~mL}$ four necked reaction vessel equipped with a reflux condenser, nitrogen gas inlet, mechanical stirrer, addition funnels, and a thermometer placed in a water bath. Solid content of the emulsion was designed as $45 \%$ by weight. Aqueous phase for emulsion polymerization was prepared by addition of D.I. Water, surfactants and buffer to the reactor (weight ratio: D.I. Water/surfactant/ $\left.\mathrm{NaHCO}_{3}=0.96 / 0.031 / 0.009\right)$, followed by the addition of the initiator (KPS, $0.8 \%$ based on total monomer mixture) at $70^{\circ} \mathrm{C}$ under nitrogen atmosphere. The polymerization was performed at $70^{\circ} \mathrm{C}$ by adding a mixture of epoxy resin dissolved in acrylate monomers (weight ratio: ER/BA/MMA/2-HEMA/AA $=0.60 / 0.22 / 0.15 / 0.015 / 0.015)$. Finally, naturalization was carried out with ethylene diamine solution and emulsion was stored in sealed container.

\subsection{Preparation of Specimen for Testing}

Specimens in the form of mild steel strips with $1000 \times$ $700 \times 0.5 \mathrm{~mm}$ size were used for analysis. Specimens were cleaned and degreased before coating application. Hybrid epoxy coatings were applied to specimen surfaces and they were left to dry for 24 hours at $28^{\circ} \mathrm{C}$. Dry film thickness was measured by a thickness gage. Two specimens were tested as soon as they were immersed in testing solution and were considered as time zero. Data were collected from specimens immersed in solution for 12 days at room temperature.

\subsection{Methods}

\section{Electrochemical Impedance Tests}

The corrosion behavior of the specimens was monitored using electrochemical impedance spectroscopy (EIS) during immersion in $3.5 \% \mathrm{NaCl}$ solution open to air and at room temperature for up to 12 days. A three-electrode set-up was used to record corrosion potential of coating. A saturated calomel electrode (SCE) was used as the reference electrode. It was coupled capacitively to a $\mathrm{Pt}$ wire to reduce the phase shift at higher frequencies. Electrochemical impedance tests were carried out by using Versa STAT 3 provided with frequency response analyzer, frequency in the range from $1 \mathrm{~Hz}$ to $1 \mathrm{kHz}$ to collect data with a total number of 40 readings for the whole range. The amplitude of the sinusoidal voltage signal was $50 \mathrm{mV}$. Data were collected by means of Frequency Response Analyzer software developed by Princeton Applied Research instruments and were in the form of Nyquist plots. Tested specimens were washed with distilled water, and then gold coated to examined under scanning electron microscope.

\subsection{Results \& Discussion}

The equivalent circuit for polymer coated steel can be represented by the model shown in Figure 1. Where, $R_{S}$ is the solution resistance, $R_{P}$ is the pore resistance of the coating, $\mathrm{R}_{\mathrm{T}}$ is the charge transfer resistance of the metal/ solution interface, $\mathrm{Cdl}$ is the double layer capacitance, and $\mathrm{Cc}$ is the coating resistance.

Figures 3-7 represent the Nyquist plots for the impedance of specimens tested after immersion in 3\% Nacl solution at room temperature for a period of 12 days. Figure 3 shows, the Nyquist plot for the specimen tested at the time of immersion, the behavior takes a shape of a part of a semicircle with high capacitive and resistive values. The overlay of bode plots for hybrid coating with different thickness are presented in the Figure 2. From which it can be predicted that hybrid coating possesses good corrosion resistance properties.

The effect of exposure time on the impedance behavior of hybrid epoxy coating can be seen from Figures 8 and 9, a severe change in capacitance and resistance values just after immersion for 4 days. Further immersion had also a great effect on the behavior of hybrid epoxy coat- ings. Immersion for 12 days and above, yielded a double semi-circle, Nyquist plot with an indication about the failure of hybrid coating and the interaction of metal surfaces with a solution. Figures $\mathbf{8}$ and $\mathbf{9}$ show the values of Capacitance (Cd) and Resistance ( $\mathrm{Rc}$ ) for specimen E as a function of immersion time. The capacitance values of the samples were very low, these values increased gradually as they were immersed in the solution. In the case of hybrid epoxy coatings, the value of $\mathrm{Cd}$ increases after

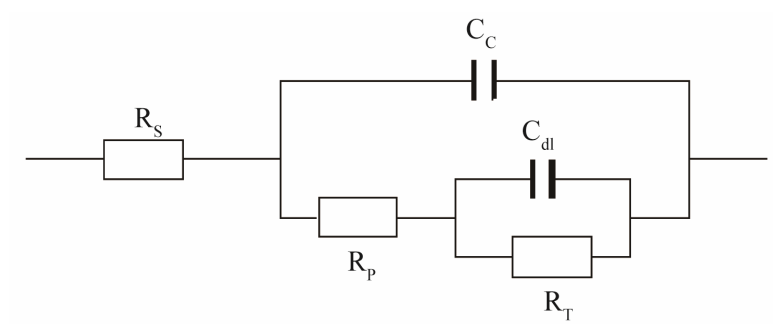

Figure 1. Equivalent RC circuit for organic coating/metal systems.

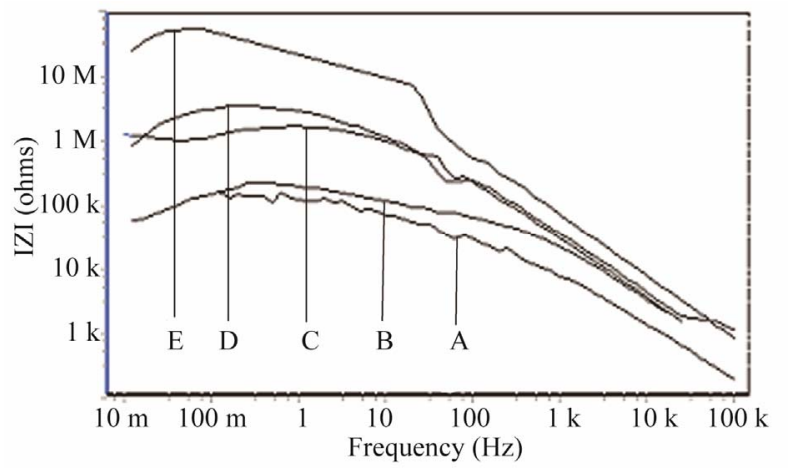

Figure 2. Overlay of Bode plot for hybrid coatings with different thickness. 


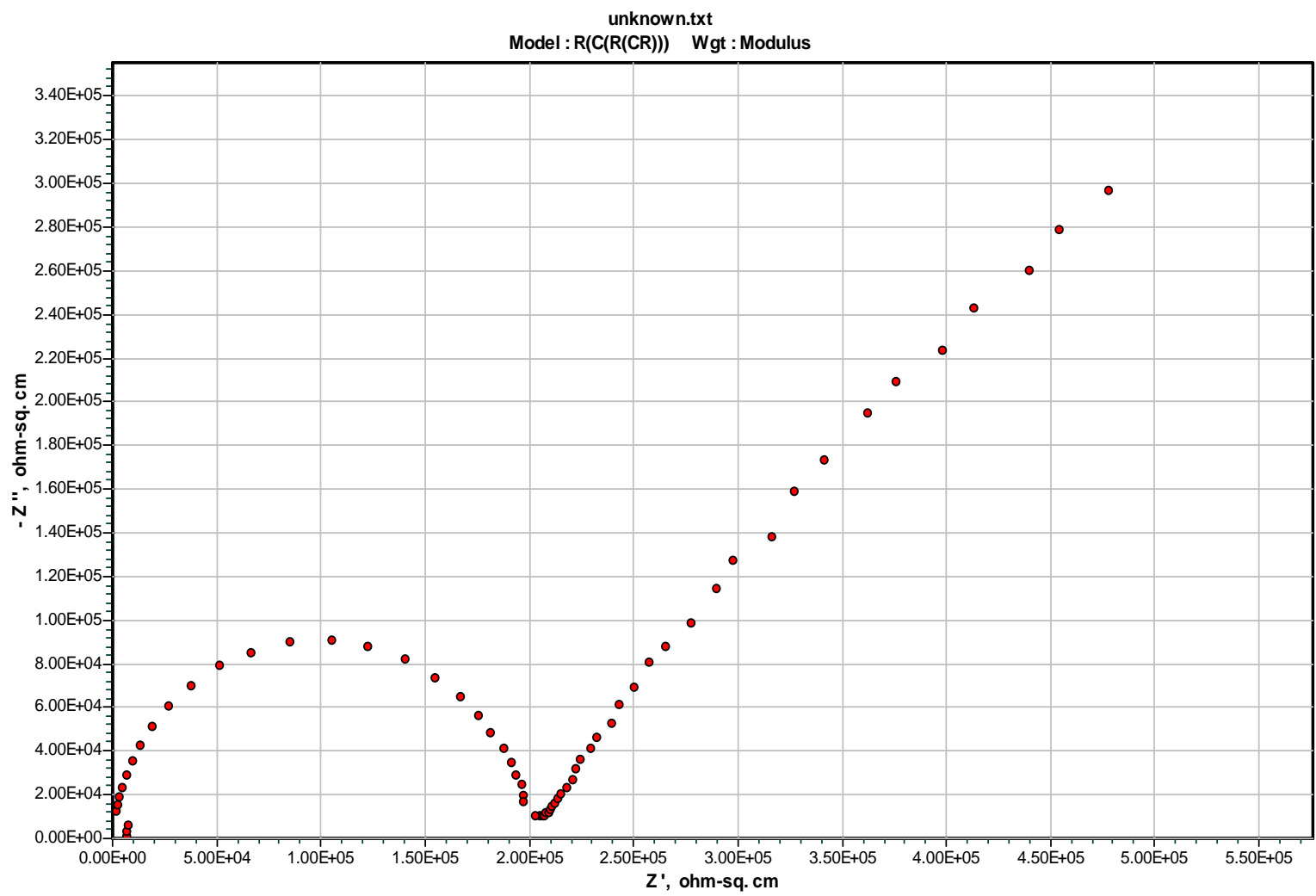

Figure 3. Nyquist plot of the epoxy-acrylate tested just after immersion in the testing solution.

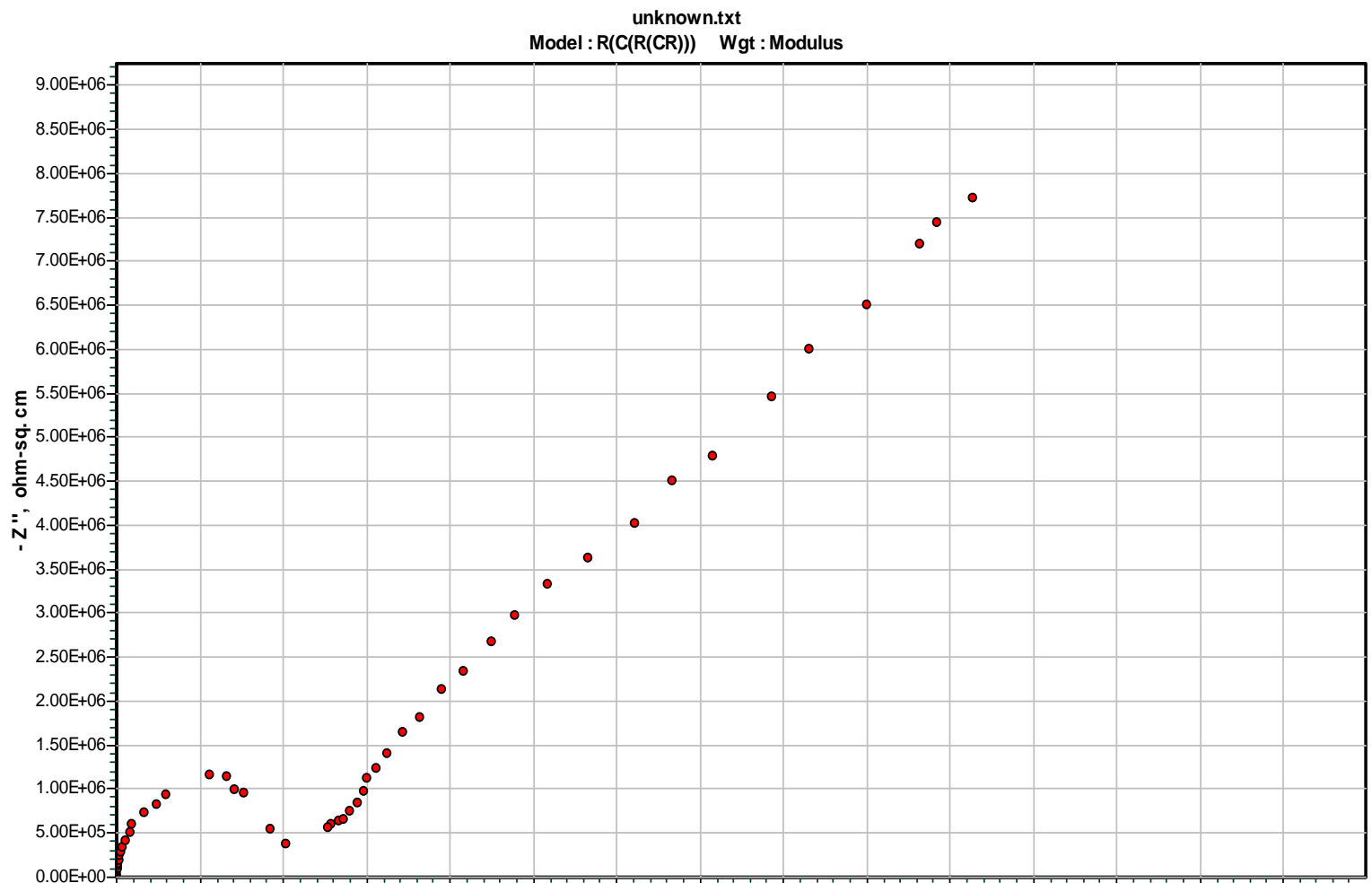

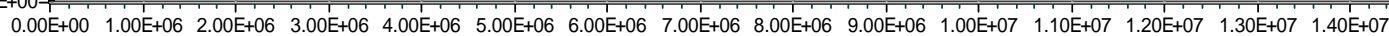
Z', ohm-sq. cm

Figure 4. Nyquist plot of the epoxy-acrylate after 4 days of immersion. 


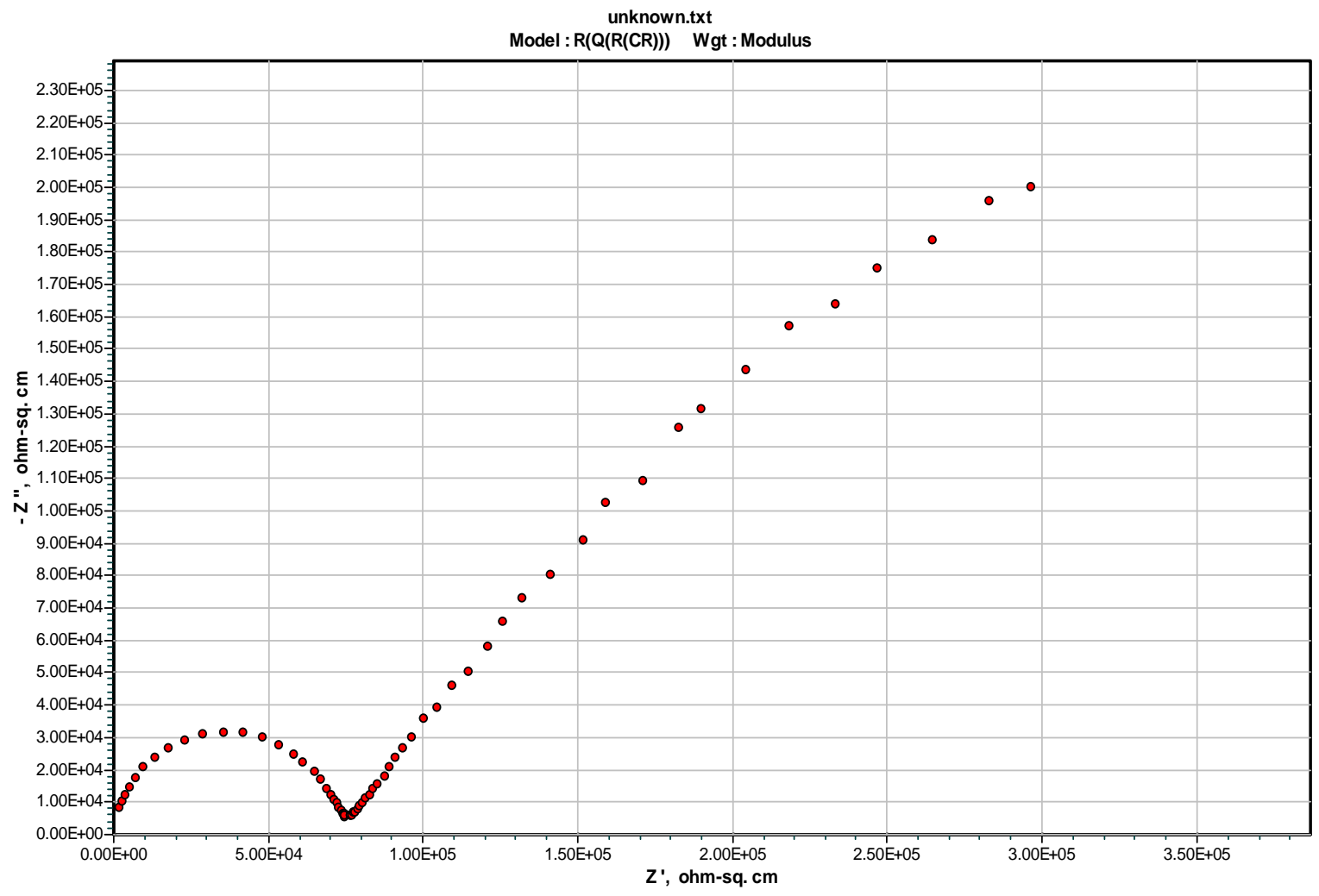

Figure 5. Nyquist plot of the sample after 8 days immersion.

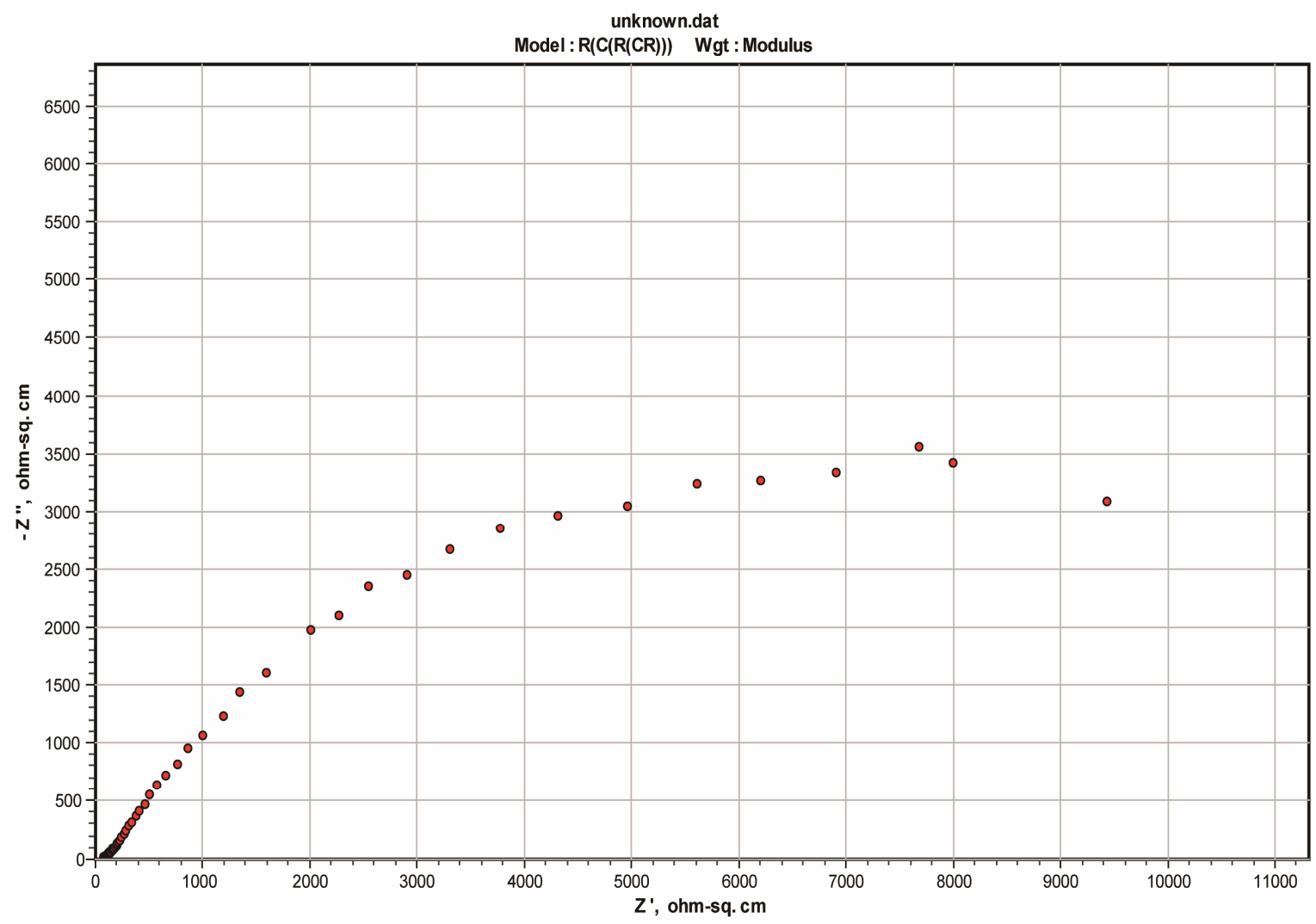

Figure 6. Nyquist plot of the sample after 10 days immersion. 


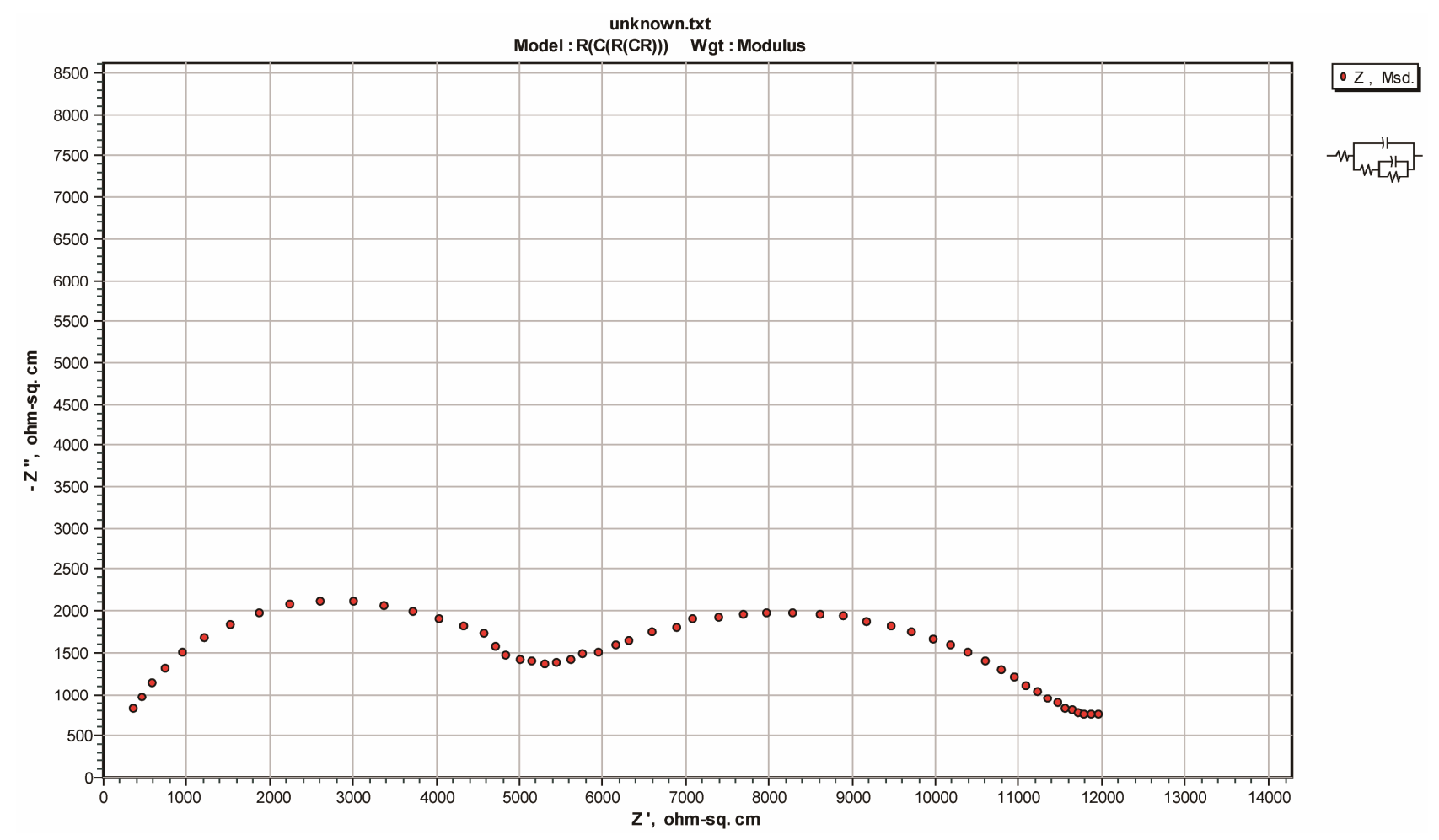

Figure 7. Nyquist plot of the sample after 12 days immersion.

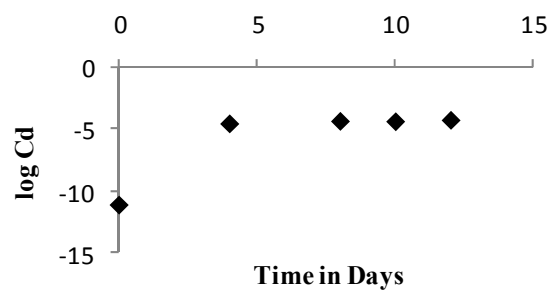

Figure 8. The value of $\mathrm{Cd}$ versus immersion time for specimen $\mathbf{E}$.

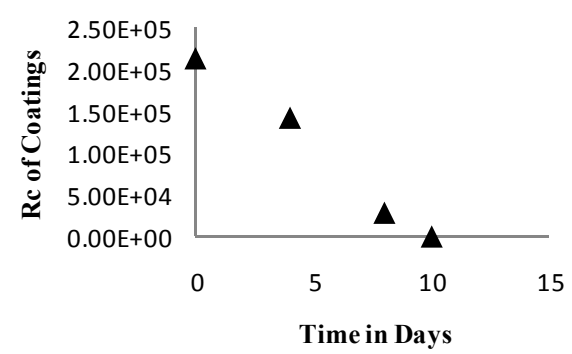

Figure 9. Re versus immersion time for specimen $\mathbf{E}$.

immersion in the electrolyte, reaching a constant value. The plateau period of $\mathrm{Cd}$ indicates the beginning of detachment of the coating from the substrate due to adhesion loss.

In the case of sample $\mathrm{E}$, a low value of $\mathrm{Cd}$ were obtained of epoxy coating just after immersion in salt water, the gradual increase in the value of $\mathrm{Cd}$ can be due to solution penetration between the coating and the steel surface. This penetration can be through breakdown sites of the coating. The high viscosity of salt water compared to water may make water uptake a time consuming process. The other parameter, $(\mathrm{Rc})$, gives an indication about the corrosion process at the steel surface. This parameter can be used to study the effectiveness of some coating in the protection of metals. Figure 9 shows the decrease in the value of Rc with respect to immersion time. Scanning Electron Micrographs taken from specimens tested after 10 days of immersion show a clear coating degradation in the form of holidays and rupture. This can be seen from Figures 13. The effect of immersion time on the performance of epoxy coating is clear, and can be seen from the values of $\mathrm{Cd}$ and $\mathrm{Rc}$.

\section{Water Absorbance Study of Hybrid Coating}

The pore resistance, $R_{P}$, which represents the ability of coating to protect the substrate, can be determined in the low frequency region in which the coating impedance is independent of frequency. The results show that hybrid coatings have their well-defined pore resistance; Table 1 summarizes the coating impedance at $0.1 \mathrm{~Hz}$ obtained in this investigation as a function of immersion time for coatings of varying thickness.

Figure 10 plots the IZI at $0.1 \mathrm{~Hz}$ as a function of coating thickness for a hybrid which are of the same coating type but at different thicknesses.

The results demonstrated that, the coating impedance increased with thickness. As shown in Figure 10, the 


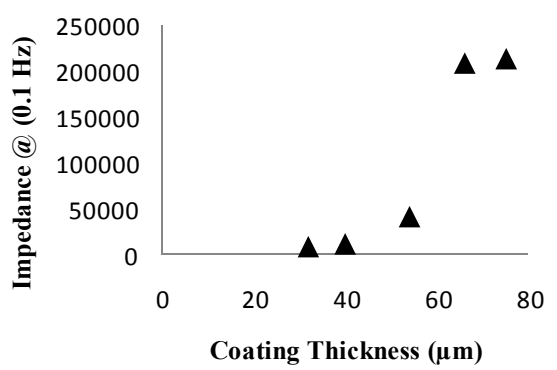

Figure 10. Plot of impedance values against coating thickness.

Table 1. Water absorption $\omega$ as a function of immersion time.

\begin{tabular}{ccccc}
\hline \multirow{2}{*}{ Specimen } & \begin{tabular}{c} 
Coating \\
thickness \\
\cline { 3 - 5 }
\end{tabular} & \multicolumn{3}{c}{ Water absorption, $\omega$} \\
\cline { 3 - 5 }$(\mu \mathrm{m})$ & 4 Day & 8 Days & 10 Day \\
\hline $\mathrm{A}$ & 32 & 0.00465 & 0.57519 & 0.62417 \\
$\mathrm{~B}$ & 40 & 0.00615 & 0.21662 & 1.18781 \\
$\mathrm{C}$ & 54 & 0.30368 & 0.59983 & 1.28901 \\
$\mathrm{D}$ & 66 & 0.15323 & 0.69160 & 1.29040 \\
$\mathrm{E}$ & 75 & 0.09285 & 0.36626 & 1.35465 \\
\hline
\end{tabular}

coating impedance at $0.1 \mathrm{~Hz}$ decreased by one order of magnitude after 8 days of immersion. Further decrease was observed during further immersion up to 12 days. During the EIS measurements, no significant corrosion or coating degradation was observed during 8 days of testing. The decreases of coating impedance during the immersion tests is most likely due to the intrusion of moisture and ions into the structures in the coatings, which in turn increased the pore conductance. This can be explained with the structural orientation of hybrid coatings during film formation.

Figure 11 demonstrates the representative FTIR spectra for the air-facing side and Fig: metal-facing side of the hybrid containing. The peak at $1508 \mathrm{~cm}^{-1}$ for stretching of paraphenyl of epoxy resin and $1729 \mathrm{~cm}^{-1}$ for the absorption of carbonyl group of acrylates. Absorbance intensity of $1508 \mathrm{~cm}^{-1}$ peak at the metal facing side is higher than those at air-facing side, suggesting that the epoxy resin part in emulsion tends to move to the metal facing side. The driving force of this movement could be attributed to the difference in the surface free energy between the epoxy resin and the acrylic copolymer. The critical surface tensions of poly butyl acrylate, polyacrylic acid, poly methyl methacrylate and poly 2 -hydoxyl ethyl methacrylate are around 31, 11.1, 39 and 37 $\mathrm{mN} / \mathrm{m}$, respectively [5], so the critical surface tension of the acrylic copolymer should be between 11 and 37 $\mathrm{mN} / \mathrm{m}$, which is lower than that of the epoxy resin, which is around $44 \mathrm{mN} / \mathrm{m}$.

Thus, during the process of casting and drying the hybrid films, the acrylic-copolymer segments tried to seg- regate near the air-facing layer and the epoxy segments moved to the mold-facing side to minimize the surface energy. This migration is very beneficial because epoxy resins have excellent adhesion to substrates improving corrosion resistance, while acrylic copolymers remaining on the air-facing side have very good weatherability and appearance.

The water absorbance of coatings due to the presence of hydrophilic acrylate monomers affects the capacitance of coatings. The measurement of the water absorption using EIS techniques is based on the determination of the changes of coating capacitance. The coating capacitance can be calculated from the EIS data $\mathrm{C}=1 / \mathrm{IZI}$ at the frequency of $1 / 2 \Pi \mathrm{Hz}$. The water absorption can be calculated by the formula given by Hartshorn et al. [6].

$$
\omega=\left[\log \left(\mathrm{C}_{\mathrm{t}} / \mathrm{C}_{0}\right)\right] /\left[\log \varepsilon_{\mathrm{H}_{2} \mathrm{O}}\right]
$$

where $\omega$ is the volume fraction of the absorbed water, $\mathrm{C}_{\mathrm{t}}$ is the coating capacitance at time $t, \mathrm{C}_{0}$ is the capacitance at $\mathrm{t}=0$, and $\varepsilon_{\mathrm{H}_{2} \mathrm{O}}$ is the dielectric constant of water. In this investigation, the coating capacitance measured immediately after immersion is taken as $\mathrm{C}_{0}$. The calculated $\omega$ using the capacitance values obtained in this work are summarized in Table 1.

The plote of water absorbance aginst immersion time for specimen A and E are shown in Figure 12. A close examination of the data in Tables 1 and $\mathbf{2}$ re- veals that correlation between IZI and $\omega$ during the im- mersion tests, indicating that the decrease of IZI was due mainly to the water absorption in the coatings. For the thin hybrid epoxy coatings tested in this investigation, the rapid water absorption in the first four days of im- mersion could be best explained by the capillary action in the micro pore/defect structures, which was followed by a slow water dissolution in the coatings. The water absorption for thicker coatings during 10 days of testing suggested that, the rates of homogeneous water dissolution into the coatings were slow. The high performance of this type of coating has been reflected by EIS data measured during 10 days of immersion. Figure 13 represents SEM micrograph for specimen after immersion test. Micrograph showing the degradation of hybrid coatings after 10 days of immersion test.

\subsection{Conclusions}

- Hybrid one pack epoxy coating was found to be effective as a protective coating against corrosion resistance.

- Corrosion of substrate by the ingress of ionic species through coating, increases disbonding between coating and substrate, which promotes the degradation of coating by the dual action of chemicals and mechanical processes. 
Table 2. Coating impedance, IZI, at $0.1 \mathrm{~Hz}$ as a function of immersion time.

\begin{tabular}{cccccc}
\hline \multirow{2}{*}{ Specimen } & \multirow{2}{*}{ Coating thickness $(\mu \mathrm{m})$} & \multicolumn{4}{c}{ Impedance $@ 0.1 \mathrm{~Hz}\left(\Omega \mathrm{cm}^{2}\right)$} \\
\cline { 3 - 6 } & & 0 Day & 4 Day & 8 Days & 10 Day \\
\hline A & 32 & $7.401841 \mathrm{e}+03$ & $7.253213 \mathrm{e}+03$ & $6.026863 \mathrm{e}+02$ & $4.867542 \mathrm{e}+02$ \\
B & 40 & $1.046800 \mathrm{e}+04$ & $1.019086 \mathrm{e}+04$ & $2.692158 \mathrm{e}+03$ & $5.894301 \mathrm{e}+01$ \\
C & 54 & $4.012673 \mathrm{e}+04$ & $1.067432 \mathrm{e}+04$ & $2.934141 \mathrm{e}+03$ & $1.453298 \mathrm{e}+02$ \\
D & 66 & $2.079440 \mathrm{e}+05$ & $1.066014 \mathrm{e}+05$ & $1.019086 \mathrm{e}+04$ & $1.067342 \mathrm{e}+03$ \\
E & 75 & $2.127438 \mathrm{e}+05$ & $1.419121 \mathrm{e}+05$ & $2.873426 \mathrm{e}+04$ & $3.860423 \mathrm{e}+02$ \\
\hline
\end{tabular}

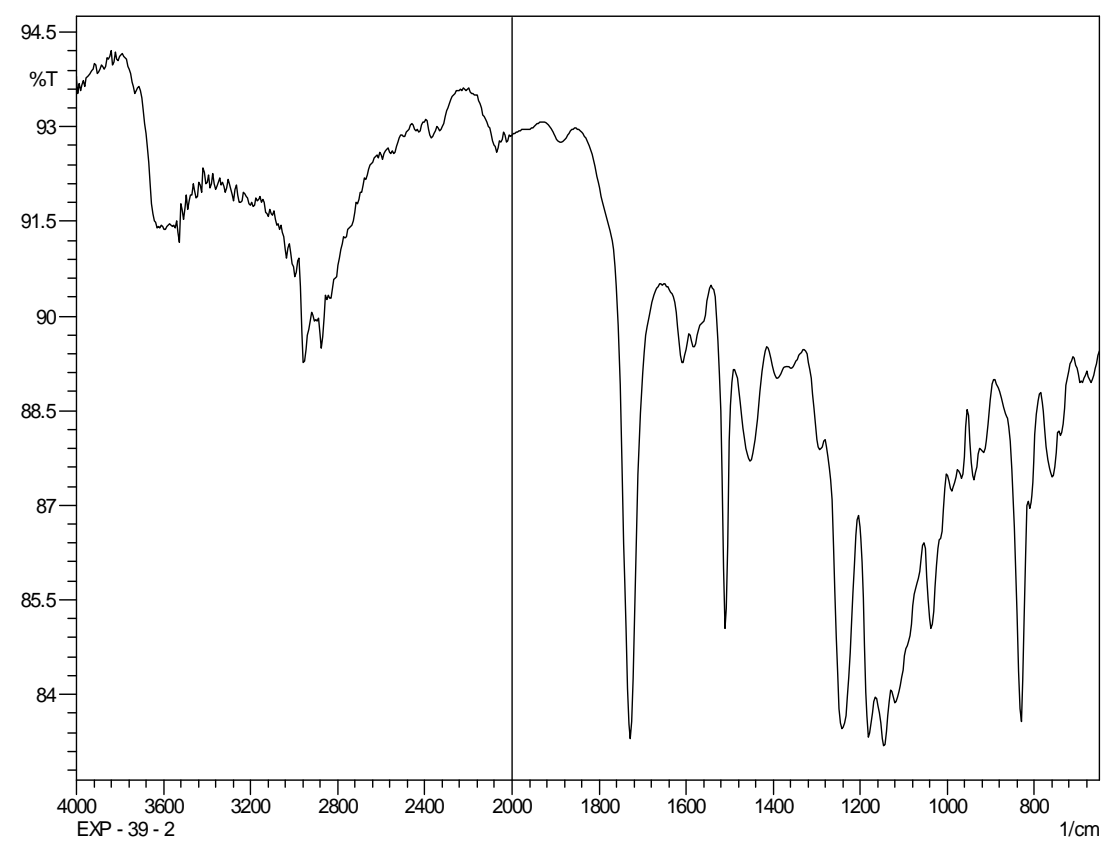

(a)

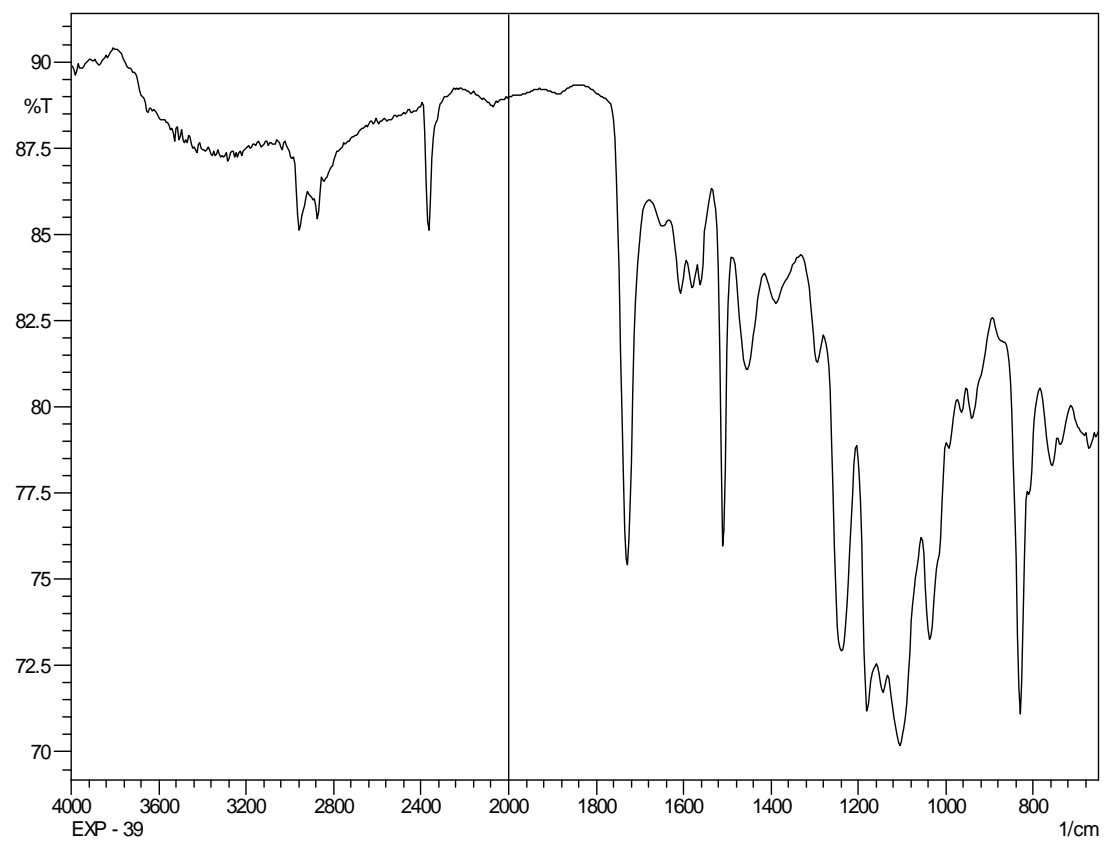

(b)

Figure 11. FTIR-ATR of hybrid (a) air facing, (b) metal facing. 


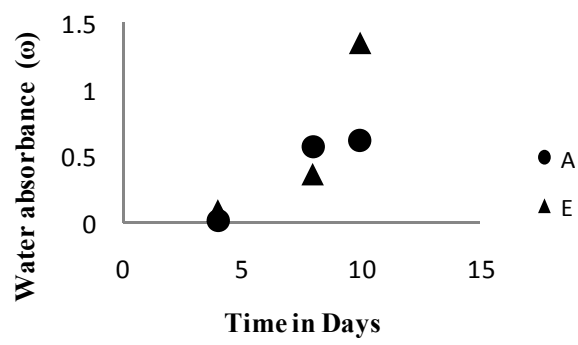

Figure 12. Plot of water absorbance $\omega$ against immersion time.

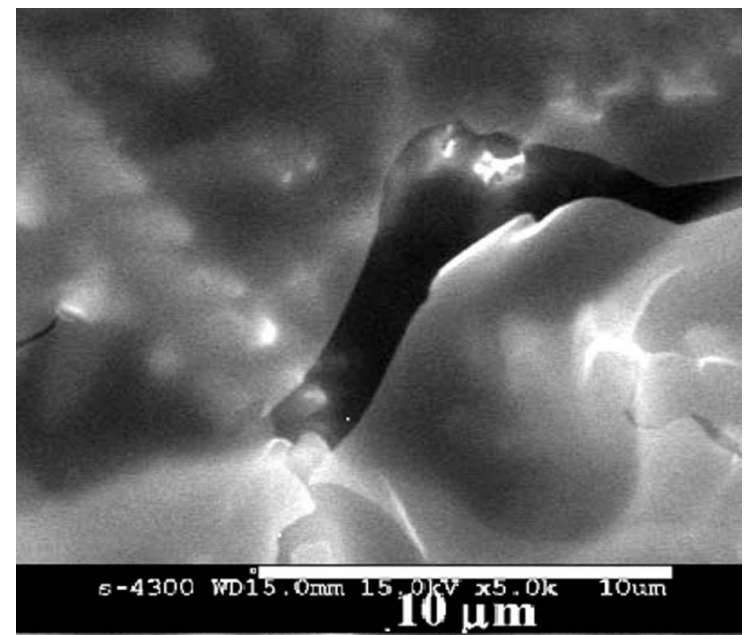

Figure 13. SEM micrograph of hybrid coating (E) after 10 days of immersion.

- For the hybrid coating impedance increased with increasing coating thickness.
- For the hybrid epoxy coatings, the quick water absorption at early immersion stage could be explained by the structure of coatings.

- It is concluded that EIS data will be useful in predicting lifetime expectancy of coatings in immersion service.

\section{REFERENCES}

[1] C. A. May, "Epoxy Resin: Chemistry and Technology," 2nd Edition, Marcel Dekker, Wiley, New York, 1988.

[2] H. Jonghun, and S. Minyoung, "Evaluation of Corrosion Protection for Epoxy and Urethane Coating by EIS under Various Cyclic Corrosion Tests," Corrosion science and Technology, Vol. 10, No. 3, 2011, pp. 95-100.

[3] X. S. Pang, G. X. Cheng and S. L. Lu, "Morphology and Thermodynamic Analysis of Composite Polymer Particles Prepared by Soap-Free Emulsion Polymerization in the Presence of Poly (Methyl Methacrylate) and Polystyrene as Bio Seeds." Journal of Applied Polymer Science, Vol. 92, No. 4, 2004, pp. 2675-2680. doi:10.1002/app.20242

[4] H. Nadia, C. Hacene, G. Gildas, B. Kamel, "The Corrosion Protection Behavior of Zinc Rich Epoxy Paint in 3\% $\mathrm{NaCl}$ Solution." Advances in Chemical Engineering and Science, Vol. 1, No. 2, 2011, pp. 51-60.

[5] J. Brandrup and E. H. Immergut, "Polymer Handbook," 2nd Edition, Wiley, New York, 1975.

[6] L. Hartshorn and E. Rushton, "Society of Chemical Industry (Great Britain),"Journal of the Indian Chemical Society, Vol. 56, No. 1, 1937, pp. 260-266. 